\title{
Magnesium Isotopes in Enamel Hydroxyapatite as Indicators of Trophic level and Digestive Strategy
}

\section{Cheng TARnG ${ }^{1 *}$ Lihai Hu ${ }^{2}$ Ogeto Mwebi ${ }^{3}$ Thure E.} CERLING $^{1}$ DIEGO P. FERNANDEZ ${ }^{1}$

${ }^{1}$ University of Utah, Department of Geology and Geophysics. *cheng.tarng@utah.edu]

${ }^{2}$ University of Ottawa, Department of Earth Sciences

${ }^{3}$ National Museum of Kenya

Over the past decades, stable isotopes of various elements preserved in fossil enamels have provided quantitative insights into past terrestrial ecological structures. Recent developments in metal isotopes geochemistry called for exploration of non-traditional isotopic systems for paleoecological applications, amoung which a multi-proxy approach to determine trophic information of the past was proposed. We report on a modern calibration of magnesium isotopic systematics with large mammalian faunas of the East African savanna ecosystems. The results of our survey show distinctive isotopic signals between carnivores and nonruminant herbivores. Furthermore, the observed magnesium isotopic signature associated with ruminants has additional potential as a proxy for digestive strategy. In sum, both findings highlight the capability and limitation of magnesium isotopes in enamel hydroxyapatite and offer promising insights for multi-proxy interpretations of past ecological systems. 